\title{
Cost Effectiveness Analysis of Filgrastim versus Placebo in Post Allogentic Bone Marrow Transplantation
}

\author{
Maoudoud Ines ${ }^{1}$, Razgallah Khrouf Myriam ${ }^{1,2}$, Ben Abdejelil Nour ${ }^{2,3}$, Ghedira Hela ${ }^{3}$, Amel Lakhal ${ }^{2,3}$, Marsit \\ Hanene $^{1}$, Turki Manel ${ }^{1}$, Soussi Mohamed Ali $^{1,2}$, Lazreg Olfa ${ }^{2}$, Ladab Saloua ${ }^{2,3}$ and Ben Othmane Tarek ${ }^{2,3}$ \\ 1. University of Monastir, Faculty of Pharmacy-5000 Tunisia \\ 2. Centre National de Greffe de Moelle Osseuse-1006 Tunisia \\ 3. University of Tunis El Manar, Faculty of Medicine of Tunis-1006 Tunisia
}

\begin{abstract}
Filgrastim is used to accelerate hematopoietic recovery after ABMT (allogeneic bone marrow transplantation). Its impact on the total cost of patient care remains to be explored. We therefore undertook a cost effectiveness analysis in the context of a randomized single blinded clinical trial of Filgrastim versus placebo in post ABMT. A primary endpoint, duration of myelosuppression, and three secondary end points (number of days of fever, length of hospital stay, survival at one hundred days) were used to assess efficacy. Direct costs were evaluated and allowed the calculation of the ICER (incremental cost-effectiveness ratios) for the major endpoint of the trial. Sixteen patients were included in the study. The duration of myelosuppression was significantly decreased in the Filgrastim arm with medians of 15 days vs. 19 days in the placebo arm $(\mathrm{p}=0.023)$. Cost analysis showed no statistically significant difference between the two arms. According to the calculation of ICER, Filgrastim was more costly and more effective than placebo for the number of days of aplasia avoided and the number of days with fever avoided. Placebo strictly dominated filgrastim for days of hospitalization avoided. Filgrastim has proven effective in reducing the duration of aplasia without increasing costs.
\end{abstract}

Key words: Filgrastim, placebo, cost, effectiveness, allogeneic bone marrow transplantation.

\section{Introduction}

ABMT (allogeneic bone marrow transplantation) remains, until the development of gene therapy, the only curative treatment of a number of constitutional deficit disorders of the hematopoietic tissue. It remains the eradicator treatment of a number of malignant hematological diseases and keeps this place in the therapeutic arsenal. However, the success of this therapy is not always guaranteed and depends on several factors such as patient age, post-transplant immunological complications, adverse reactions of the conditioning treatment, the occurrence of infections associated with neutropenia caused by myeloablation and / or myelosuppression and the duration of the neutropenia [1].

The Filgrastim, a granulocyte colony-stimulating

Corresponding author: Maoudoud Ines, Pharm. D, resident in medical biology (subspeciality immunology). factor analog, is used to reduce the duration of neutropenia in patients undergoing myeloablative therapy followed by bone marrow transplantation [2]. Hence it represents an additional cost of drug spending in the care of patients undergoing ABMT. Widespread use should be based on a rational assessment of cost effectiveness in a context where health expenditures are increasing [3].

We intend to conduct a cost-effectiveness analysis of Filgrastim (Neupogen ${ }^{\circledR}$ ) vs. placebo in reducing the duration of neutropenia in patients undergoing myeloablative therapy followed by ABMT.

\section{Patients and Methods}

This pilot study is part of the evaluation of the effectiveness and cost of Filgrastim after geno-identical ABMT. It concerns adult patients hospitalized in the sterile unit of the Hematology / Bone Marrow Transplantation service in the Centre 
National de Greffe de Moelle Osseuse-Tunisia. Patients were randomized to one of two groups (Filgrastim or placebo) in a single blinded conducted trial. We had the agreement of the ethics committee for the conduct of this trial.

Filgrastim is used in primary prevention on the regimen of $5 \mu \mathrm{g} / \mathrm{Kg}$ once daily by intravenous infusion. The administration begins on day 7 post ABMT and is maintained 72 hours after the ANC (absolute neutrophil count) stabilizes above 1000 elements/ $\mu \mathrm{L}$. Patients of the placebo group were given only saline by the same regimen.

Data collection has been done by means of a table including patient characteristics and different clinical parameters used to assess efficacy. The study covered a period of one hundred days from the day of the transplantation.

\subsection{Effectiveness}

The primary endpoint was the myelosuppression period defined by an $\mathrm{ANC}<500 / \mu \mathrm{L}$. The secondary endpoints were the number of days of fever, the number of days of hospital stay and survival at 100 days.

\subsection{Cost Data}

Direct costs were calculated for both arms of the study and are expressed in Euros. It includes cost of laboratory tests, cost of medical imaging, drug costs (divided into anti-infectives, parenteral nutrition, oral decontamination, Filgrastim and other medicines), cost of TDM (therapeutic drug monitoring) and cost of labile blood products.

\subsection{Cost-Effectiveness Analysis}

ICER (incremental cost-effectiveness ratios) expressing the additional cost of one unit of outcome gained/avoided by one strategy compared with another, were calculated for the mainly endpoints of the trial. It is the ratio of the difference in costs between the two arms of treatment (Filgrastim and placebo) to the difference in effectiveness [4].

\subsection{Statistical Analysis}

A statistical analysis was performed through SPSS (Statistical Package for the Social Sciences) Version 21.

Quantitative variables were described as median, mean and standard deviation. Qualitative variables were described as percentages.

The efficacy endpoints and the different costs were compared between the two independent samples (Filgrastilm arm and placebo arm). Quantitative variables were analyzed using the Mann-Witheney $U$ test. The Fisher exact test was used to compare categorical variables. The difference was considered statistically significant when the $p$ value was less than 0.05 in a bi-tailed test.

\section{Results}

\subsection{Patient Characteristics}

A total of sixteen patients (nine in the Filgrastim arm and seven in the placebo arm) aged 17 to 37 years (Table 1) participated in the study. All underwent geno-identical ABMT and received a GvHD prophylaxis based on ciclosporin and short methotrexate.

\subsection{Effectiveness}

The duration of myelosuppression was shorter in the Filgrastim arm relative to the placebo arm (median of 15 days vs. 19 days) $(p=0.023)$ (Table 2$)$.

\subsection{Cost Data}

The total cost was higher in the Filgrastim arm but the difference between the two groups was not significant (Table 3 ).

\subsection{Cost Effectiveness Analysis}

The calculation of ICER (Table 4) showed that the amount to be paid to avoid a day of myelosuppression was $260 €$. The amount to be paid to avoid a day of 
fever was $866.2 €$.

The Filgrastim was less effective and more expensive than placebo in reducing the number of days of hospitalisation (ICER $=-600$ ) as shown in Table 4 .

Table 1 Patient characteristics.

\begin{tabular}{|c|c|c|c|}
\hline & Filgrastim $(\mathrm{N}=9)$ & Placebo $(\mathrm{N}=7)$ & $p$ \\
\hline $\operatorname{Age}^{a}$ (years) & $24.78 \pm 7.31$ & $25 \pm 6.53$ & 0.98 \\
\hline Sex ratio (males : females) & $4: 5$ & $6: 1$ & 0.15 \\
\hline Body Mass Index $\left(\mathrm{Kg} / \mathrm{m}^{2}\right)^{\mathrm{a}}$ & $20.16 \pm 1.88$ & $24.86 \pm 5.21$ & 0.02 \\
\hline \multicolumn{4}{|l|}{ Diagnosis ${ }^{b}$} \\
\hline Acute leukemia & $5(55.6 \%)$ & $2(28.6 \%)$ & \multirow{3}{*}{0.36} \\
\hline Aplastic anemia & $4(44.4 \%)$ & $5(71.4 \%)$ & \\
\hline Standard risk ${ }^{\mathrm{b}}$ & $7(77.8 \%)$ & $6(85.7 \%)$ & \\
\hline High risk $^{\mathrm{b}}$ & $2(22.2 \%)$ & $1(14.3 \%)$ & 1 \\
\hline \multicolumn{4}{|l|}{ Sex Mismatch } \\
\hline Present ${ }^{b}$ & $6(66.6 \%)$ & $3(42.9 \%)$ & \multirow{3}{*}{0.62} \\
\hline Absent $^{\mathrm{b}}$ & $3(33.3 \%)$ & $4(57.1 \%)$ & \\
\hline \multicolumn{3}{|l|}{ ABO compatibility } & \\
\hline Compatible $^{\mathrm{b}}$ & $7(77.8 \%)$ & $2(28.6 \%)$ & \multirow{5}{*}{0.13} \\
\hline Incompatible $^{b}$ & $2(22.2 \%)$ & $5(71.4 \%)$ & \\
\hline Major & 1 & 2 & \\
\hline Minor & 1 & 1 & \\
\hline Mixed & 0 & 1 & \\
\hline Mononuclear cells infused $\left(\times 10^{8} / \mathrm{kg}\right)^{\text {a }}$ & $1.89 \pm 0.59$ & $2 \pm 0.75$ & 0.78 \\
\hline
\end{tabular}

${ }^{a}$ mean \pm standard deviation.

${ }^{b} \mathrm{n}(\%)$.

Table 2 Efficiency measured according to the treatment group.

\begin{tabular}{|c|c|c|c|c|c|c|c|}
\hline \multirow{2}{*}{ Endpoints } & \multicolumn{3}{|c|}{ Filgrastim $(\mathrm{N}=9)$} & \multicolumn{3}{|c|}{ Placebo $(\mathrm{N}=7)$} & \multirow{2}{*}{$p$} \\
\hline & Median & Mean & Standard deviation & Median & Mean & Standard deviation & \\
\hline $\begin{array}{l}\text { Duration of } \\
\text { myelosuppression* }\end{array}$ & 15 & 15.44 & 1.24 & 19 & 19.57 & 4.39 & 0.03 \\
\hline Number of days of fever & 4 & 6.33 & 5.70 & 4 & 7.57 & 6.85 & 0.90 \\
\hline Duration of hospitalization* & 24 & 26.22 & 6.78 & 25 & 24.43 & 4.86 & 0.98 \\
\hline Survival at 100 days* & 100 & 100 & 0 & 100 & 87.85 & 32.13 & 0.44 \\
\hline
\end{tabular}

*days.

Table 3 Mean total costs per patient (in 2015 euros).

\begin{tabular}{|c|c|c|c|c|c|}
\hline \multirow{2}{*}{ Costs } & \multicolumn{2}{|c|}{ Filgrastim $(\mathrm{N}=9)$} & \multicolumn{2}{|c|}{ Placebo $(\mathrm{N}=7)$} & \multirow{2}{*}{$p$} \\
\hline & Mean & $\overline{\text { Standard deviation }}$ & Mean & Standard deviation & \\
\hline Anti infectives & 2347.8 & 1098.5 & 3880.5 & 1783.0 & 0.83 \\
\hline Filgrastim & 1753.2 & 254.1 & 0.0 & 0.0 & $<10^{-4}$ \\
\hline Parenteral Nutrition & 321.1 & 147.9 & 262.4 & 120.5 & 0.75 \\
\hline Oral Decontamination & 91.8 & 85.3 & 79.9 & 36.7 & 0.75 \\
\hline Other medicines & 608.8 & 160.0 & 1149.9 & 528.3 & 1 \\
\hline Total (medicines) & 5122.8 & 1051.3 & 5372.5 & 2468.5 & 0.53 \\
\hline Therapeutic Drug Monitoring & 517.0 & 70.7 & 397.0 & 182.4 & 0.12 \\
\hline Blood Derivatives & 873.0 & 361.9 & 1016.6 & 467.1 & 1.00 \\
\hline Biological analyzes & 3387.9 & 1802.1 & 2138.3 & 982.5 & 0.09 \\
\hline $\begin{array}{l}\text { Medical imaging and } \\
\text { anatomopathological analyzes }\end{array}$ & 170.8 & 260.3 & 73.1 & 33.6 & 0.45 \\
\hline Total & 10071.6 & 2145.4 & 8997.5 & 4134.1 & 0.35 \\
\hline
\end{tabular}


Allogentic Bone Marrow Transplantation

Table 4 Incremental cost-effectiveness ratios (ICERs) of Filgrastim versus placebo.

\begin{tabular}{lllll}
\hline & Filgrastim & Placebo & difference & ICER \\
\hline Mean cost $(€, 2015)$ & 10071,6 & 8997.5 & 1074.1 & \\
Effects : mean days & & & & \\
Myelosuppression & 15.44 & 19.57 & 4.13 & 260.1 \\
Fever & 6.33 & 7.57 & 1.24 & 866.2 \\
Hospitalisation & 26.22 & 24.43 & -1.79 & -600.1 \\
\hline
\end{tabular}

\section{Discussion}

Our study shows that filgrastim reduces the duration of myelosuppression from 19 days (placebo group) to 15 days $(p=0.023)$ as shown in Table 2. This was demonstrated in two other studies by Bishop et al and Ernst et al (11 days and 15 days repectively for Filgrastim and 15 days and 19 days respectively for placebo $[5,6]$.

In this study, the acceleration of neutrophil engraftment by Filgrastim did not affect significantly 100 day survival. This was also observed in the two studies cited above $[5,6]$. Due to some controversies, the real impact on survival of G-CSF remains unclear $[7,8]$.

The Filgrastim does not constitute an additional expense compared to placebo according to the costs measured in our study (Table 3 ). The ICER in reducing myelosuppression is about 260 Euros. This should be compared to the threshold values of ICER depending on social, economical and political factors. Several alternatives seeking to improve the cost-effectiveness ratio of myeloid growth factors used pegfilgrastim (a covalent conjugate of Filgrastim characterized by a greater half-life [7] and found it more efficient and cheaper [9]. The pegfilgrastim has also been proposed as an alternative in some of the recommendations to Filgrastim $[2,7]$. The cost-effectiveness of G-CSF mimetics (second generation products) remains to be explored.

This study presents multiple bias. It is based on a reduced cohort of sixteen patients. This pilot study should be continued with a larger number of patients. The body mass index was significantly different between the two arms of treatment. This could be a source of bias such in the assessment of the cost of medicines or if it causes some comorbidities.

\section{Conclusion}

The Filgrastim shows an interesting effectiveness in reducing the duration of myelosuppression in allograft patients, without any significant change in the number of days of fever, length of hospital stay or survival. It does not present a significant additional cost in return compared to placebo. Larger studies are needed to confirm our results. We mention that the cost effectiveness of filgrastim may be significantly improved by the adoption of biosimilars.

\section{References}

[1] Gratwohl, A., Hermans, J., Goldman, J. M., Arcese, W., Carreras, E., Devergie, A., et al. 1998. "Risk Assessment for Patients with Chronic Myeloid Leukaemia before Allogeneic Blood or Marrow Transplantation. Chronic Leukemia Working Party of the European Group for Blood and Marrow Transplantation." Lancet 352 (9134): 1087-92.

[2] Crawford, J., Armitage, J., Balducci, L., Becker, P. S., Blayney, D. W., Cataland, S. R., et al. 2013. "Myeloid Growth Factors." Journal of the National Comprehensive Cancer Network: JNCCN 11 (10): 1266-90.

[3] Hirsch, B. R., and Lyman, G. H. 2012. "Pharmacoeconomics of the Myeloid Growth Factors: A Critical and Systematic Review." Pharmaco Economics 30 (6): 497-511.

[4] Bang, H., and Zhao, H. 2012. "Median-Based Incremental Cost-Effectiveness Ratio (ICER)." Journal of Statistical Theory and Practice 6 (3): 428-42.

[5] Ernst, P., Bacigalupo, A., Ringden, O., Ruutu, T., Kolb, H. J., Lawrinson, S., et al. 2008. "A Phase 3, Randomized, Placebo-controlled Trial of Filgrastim in Patients with Haematological Malignancies Undergoing Matched-related Allogeneic Bone Marrow Transplantation." Archives of Drug Information 1 (3): 89-96.

[6] Bishop, M. R., Tarantolo, S. R., Geller, R. B., Lynch, J. C., Bierman, P. J., Pavletic, Z. S., Vose, J. M., Kruse, S., Dix, 
S. P., Morris, M. E., Armitage, J. O., and Kessinger, A. 2000. "A Randomized, Double-Blind Trial of Filgrastim (Granulocyte Colony-Stimulating Factor) versus Placebo Following Allogeneic Blood Stem Cell Transplantation.” Blood 96: 6.

[7] Hoggatt, J., and Pelus, L. M. 2014. "New G-CSF Agonists for Neutropenia Therapy." Expert Opinion on Investigational Drugs 23 (1): 21-35.

[8] Ringden, O., Labopin, M., Gorin, N. C., Le Blanc, K., Rocha, V., Gluckman, E., et al. 2004. "Treatment with Granulocyte Colony-Stimulating Factor after Allogeneic Bone Marrow Transplantation for Acute Leukemia Increases the Risk of Graft-Versus-Host Disease and
Death: A Study from the Acute Leukemia Working Party of the European Group for Blood and Marrow Transplantation." Journal of Clinical Oncology: Official Journal of the American Society of Clinical Oncology 22 (3): 416-23.

[9] Perrier, L., Lefranc, A., Perol, D., Quittet, P., Schmidt-Tanguy, A., Siani, C., et al. 2013. "Cost Effectiveness of Pegfilgrastim versus Filgrastim after High-Dose Chemotherapy and Autologous Stem Cell Transplantation in Patients with Lymphoma and Myeloma: An Economic Evaluation of the PALM Trial." Applied Health Economics and Health Policy 11 (2): 129-38. 